\title{
ENSINO DA AVALIAÇÃO NOS CURSOS DE EDUCAÇÃO FÍSICA DA AMÉRICA LATINA
}

\section{SAYONARA CUNHA DE PAULA' AMARÍLIO FERREIRA NETO" RONILDO STIEG"I WAGNER DOS SANTOSIV}

\section{RESUMO}

Este artigo analisa como a avaliação do ensino e da aprendizagem é prescrita nos currículos dos cursos de formação de professores em Educação Física em sete países da América Latina. Caracteriza-se como pesquisa qualitativa, do tipo exploratória, que assume a análise crítico-documental como abordagem teórico-metodológica e o Interface de $\mathrm{R}$ pour les Analyses Multidimensionnelles de Textes e de Questionnaires (IRaMuTeQ) como ferramenta de auxílio para análise. Utiliza, como fontes, 14 planos de disciplinas de avaliação. A análise dos dados indica que as disciplinas se propõem a discutir e ensinar a avaliação do processo de ensino e da aprendizagem,

I Universidade Federal do Espírito Santo (UFES) Vitória-ES, Brasil; sayocpaula@hotmail.com

II Universidade Federal do Espírito Santo (UFES), Vitória-ES Brasil; amariliovix@gmail.com

III Universidade Federal do Espirito Santo (UFES), Vitória-ES, Brasil; roni.stieg@gmail.com

IV Universidade Federal do Espírito Santo (UFES), Vitória-ES

Brasil;wagnercefd@gmail.com suas implicações para o contexto da educação básica, projeção de possibilidades de práticas avaliativas e estabelece a relação teoria e prática. Esse movimento evidencia-se com a presença de algumas palavras, como "instrumento", "técnica", "prática", articulando-se também com a "teoria e a concepção de avaliação" que the oferece suporte, relacionando-se com a "Educação Física" no contexto da educação profissional. 


\title{
ENSEÑANZA DE LA EVALUACIÓN EN LOS CURSOS DE EDUCACIÓN FÍSICA DE AMÉRICA LATINA
}

RESUMEN

Este artículo analiza cómo la evaluación de la enseñanza y del aprendizaje se prescribe en los currículos de los cursos de formación de profesores en Educación Física en siete países de América Latina. Se caracteriza como investigación cualitativa, del tipo exploratoria, que asume el análisis crítico-documental como abordaje teórico-metodológico y lo Interface de R pour les Analyses Multidimensionnelles de Textes e de Questionnaires (IRaMuTeQ) como herramienta auxiliar para el análisis. Utiliza, como fuentes, 14 planos de disciplinas de evaluación. El análisis de los datos indica que las asignaturas se proponen a discutir y enseñar la evaluación del proceso de enseñanza y aprendizaje, sus implicaciones para el contexto de la educación básica, proyección de posibilidades de prácticas evaluativas y establece la relación entre teoría y práctica. Este movimiento se pone de manifiesto con la presencia de algunas palabras, como "instrumento", "técnica", "práctica", articulándose también con la "teoría y la concepción de evaluación" que le ofrece soporte, relacionándose con la "Educación Física” en el marco de la educación profesional.

PALABRAS CLAVE EVALUACIÓN DE LA EDUCACIÓN • EDUCACIÓN FÍSICA • FORMACIÓN DE PROFESORES • CURRÍCULO.

\section{TEACHING EVALUATION IN PHYSICAL EDUCATION COURSES IN LATIN AMERICA}

\begin{abstract}
This article looks at how the evaluation of teaching and learning is prescribed in the curricula of teacher training courses in Physical Education in seven countries of Latin America. It is a qualitative, exploratory study, which uses critical documentary analysis as a theoretical-methodological approach and the Interface de R pour les Analyses Multidimensionnelles de Textes et de Questionnaires (IRaMuTeQ) as a tool to support analysis. Fourteen subject evaluation plans are used as sources. The analysis of the data indicates that the subjects are intended for discussion and teaching of evaluation of the teaching and learning processes, their implications in the context of basic education, the projection of possibilities of evaluation practices and for establishing the relationship between theory and practice. This trend becomes clear through the use of some words, such as "tool", "technique", "practice", associated with the "theory and design of evaluation" that supports it, relating to "physical education" in the context of professional education.

KEYWORDS EVALUATION OF EDUCATION • PHYSICAL EDUCATION • TEACHER TRAINING • CURRICULUM.
\end{abstract}


1 “[...] tipo de prescrição ou orientação do que deve ser seu conteúdo, principalmente em relação à escolaridade obrigatória. [...] aspectos que atuam como referência na ordenação do sistema curricular servem de ponto de partida para a elaboração de materiais, controle do sistema, etc." (p. 104)

2 "Por avaliação do ensino e da aprendizagem entendemos que essa ocorre no contexto da sala de aula. envolvendo o professor e o aluno, permitindo evidenciar

o processo educativo oferecendo pistas do que o estudante aprendeu" (p. 47)

\section{INTRODUÇÃO}

Como a avaliação do ensino e da aprendizagem é prescrita nos currículos (SACRISTÁN, 2000) ${ }^{1}$ dos cursos de formação de professores em Educação Física nos países da América Latina? Esse questionamento gera o objetivo deste estudo, que é compreender como a avaliação do ensino e da aprendizagem (FREITAS, 2014)² é prescrita nas disciplinas específicas sobre o tema nos cursos de formação de professores em Educação Física nos países da América Latina.

A articulação entre a avaliação e a formação de professores tem sido objeto de estudo de alguns pesquisadores do campo da Educação Física em países da América Latina (BATISTA, 2000; FROSSARD, 2015; GALLARDO-FUENTES; LÓPEZ-PASTOR; CARTER-THUILLIER, 2017; MENDES; NASCIMENTO; MENDES JÚNIOR, 2007; SANTOS et al., 2016b; SARNI, 2006). Esses autores sinalizam a necessidade de maior discussão sobre essa temática, destacando sua relevância no exercício da docência.

No Uruguai, Sarni (2006) investigou as concepções de avaliação da aprendizagem presentes nos discursos e práticas de professores de Educação Física da rede pública de Montevidéu 
e sua possível relação com os currículos de formação dos docentes, em particular da disciplina de avaliação. Os resultados apontam que as práticas de avaliação da Educação Física escolar não estão relacionadas aos interesses presentes nos programas de disciplinas sinalizados.

No Brasil, Santos et al. (2016b), ao investigarem o modo como os alunos do último período do curso de licenciatura do Centro de Educação Física e Desportos da Universidade Federal do Espírito Santo (CEFD/UFES) (res)significam suas experiências com a avaliação do processo de ensino-aprendizagem vivenciada na formação inicial, ressaltam que as narrativas dos discentes centralizam-se nas práticas realizadas por seus professores e, por se colocarem na condição de alunos, focalizam a maneira pela qual são avaliados.

Os autores sinalizam a necessidade de provocar uma reflexão sobre a atuação profissional e de aprofundar a discussão entre formação inicial e avaliação, problematizando a própria compreensão desses conceitos. Além disso, apontam que a articulação das experiências avaliativas com a futura atuação docente apresenta-se especialmente em disciplinas que discutem as práticas pedagógicas, como o Estágio Supervisionado.

Nesse mesmo contexto, Batista (2000), Mendes, Nascimento e Mendes Júnior (2007) e Frossard (2015) também enfatizam a necessidade de as diferentes disciplinas que constituem o currículo de formação inicial discutirem sobre avaliação, uma vez que esse é um anseio anunciado pelos alunos participantes dos estudos.

No Chile, Gallardo-Fuentes, López-Pastor e Carter-Thuillier (2017) investigaram as práticas de avaliação formativa usadas no processo de formação inicial em Educação Física, comparando a percepção de alunos, professores e egressos. Os autores assim evidenciam: existe uma alta consistência interna entre os programas das disciplinas e sistemas de avaliação realmente utilizados; as técnicas e instrumentos de avaliação direcionados para o sistema de avaliação formativa; e há uma baixa participação dos alunos na avaliação; por fim, sinalizam a necessidade de pesquisas sobre a temática em mais universidades do país. 
3 Utilizamos, neste estudo, o termo plano de disciplina para uniformizar a escrita do texto. Geralmente é organizado em dados de identificação da disciplina, ementa, objetivos, conteúdo programático, metodologia avaliação e bibliografia básica e complementar da disciplina.

4 Pelo projeto da Universidade Federal de Goiás (UFG), Análise comparativa do perfil da formação profissional em educação física: a América Latina em foco, em que os dados foram provenientes dos websites das instituições e posteriormente, organizados em pastas separadas por país, contendo legislações currículos das instituições.

5 Assumimos as siglas no intuito de padronizar a abreviação das instituições tomando como referência o modo como algumas o fazem em seu endereço eletrônico.

\section{TEORIA E MÉTODO}

Caracteriza-se como pesquisa qualitativa exploratória que assume a análise crítico-documental (BLOCH, 2001) como abordagem teórico-metodológica. Utiliza, como fonte, os currículos prescritos (SACRISTÁN, 2000) dos cursos de formação de professores em Educação Física de sete países hispanofalantes da América Latina.

Nesse sentido, analisamos os planos de disciplinas ${ }^{3}$ de instituições de ensino latino-americanas, entendendo que esse documento define e organiza o que será ensinado. Buscamos, nesse documento, compreender o ensino da avaliação para o professor de Educação Física nesses países.

Com base no banco de dados compartilhado, ${ }^{4}$ analisamos o material e estabelecemos os seguintes critérios para delimitação das fontes: a) ter em seu currículo uma disciplina específica e obrigatória sobre avaliação educacional; e b) ser qualquer país da América Latina de idioma hispanofalante. Dos 13 países latino-americanos presentes no banco de dados, quatro não atenderam ao critério $a$ e um ao critério $b$ de nossa pesquisa, sendo assim composta pela Argentina, Chile, Colômbia, Equador, México, Uruguai e Venezuela.

Para essa parte do estudo, analisamos, inicialmente, 38 instituições de formação de professores dos respectivos países que apresentam, em seus currículos, disciplinas específicas de avaliação educacional.

Após a definição das instituições, encaminhamos um e-mail com ofício, explicando os objetivos e finalidades da pesquisa, esclarecendo possíveis dúvidas e convidando-os para o estudo. Também solicitamos o envio do plano de disciplina. Esse processo ocorreu entre janeiro e abril de 2017. Dos 38 contatos realizados, 13 instituições nos enviaram os planos: duas da Argentina, quatro do Chile, duas da Colômbia, uma do Equador, uma do México, uma do Uruguai e duas da Venezuela. Para a apresentação das disciplinas, elaboramos o Quadro 1, do qual constam os países e nomes das instituições com as respectivas siglas. ${ }^{5}$ 
QUADRO 1 - Relação das instituições e disciplinas

\begin{tabular}{|c|c|c|}
\hline $\begin{array}{l}\text { PAís } \\
(N=7)\end{array}$ & UNIVERSIDADE/INSTITUIÇÃO (N=13) & $\begin{array}{l}\text { DISCIPLINA } \\
(N=14)\end{array}$ \\
\hline \multirow[t]{2}{*}{ Argentina (ARG) } & $\begin{array}{l}\text { Instituto Superior de Educación Física } \\
\text { Federico Williams Dickens (ISEFFD/ARG) }\end{array}$ & Evaluación Aplicada \\
\hline & Universidad Maimónides (UM/ARG) & Metodología de la Evaluación y Estadistica Aplicada \\
\hline \multirow[t]{5}{*}{ Chile (CHL) } & Universidad de Concepción (UDEC/CHL) & Evaluación de Aprendizaje \\
\hline & \multirow[t]{2}{*}{ Universidad de Atacama (UDA/CHL) } & Metodología y Evaluación de la Educación Física \\
\hline & & Evaluación Educacional \\
\hline & Universidad Andrés Bello (UNAB/CHL) & Currículum y Evaluación \\
\hline & $\begin{array}{l}\text { Universidad Adventista de Chile (UNACH/ } \\
\mathrm{CHL} \text { ) }\end{array}$ & Evaluación Educacional \\
\hline \multirow[t]{2}{*}{ Colômbia (COL) } & Universidad Católica de Oriente (UCO/COL) & Teoría de la Evaluación Escolar \\
\hline & Institución Universitaria (CESMAG/COL) & Evaluación Educativa \\
\hline Equador (ECU) & Universidad Nacional de Loja (UNL/ECU) & Evaluación Curricular \\
\hline México (MEX) & $\begin{array}{l}\text { Universidad Autónoma de Baja California } \\
\text { (UABC/MEX) }\end{array}$ & Evaluación de la Actividad Física y Deporte \\
\hline Uruguai (URY) & Universidad de la República (UDELAR/URY) & Evaluación \\
\hline \multirow[t]{2}{*}{ Venezuela (VEN) } & $\begin{array}{l}\text { Universidad Nacional Experimental de } \\
\text { los Llanos Occidentales Ezequiel Zamora } \\
\text { (UNELLEZ/VEN) }\end{array}$ & Evaluación del Aprendizaje \\
\hline & $\begin{array}{l}\text { Universidad Nacional Experimental de } \\
\text { Guayana (UNEG/VEN) }\end{array}$ & Evaluación de los Aprendizajes \\
\hline
\end{tabular}

Fonte: Elaboração dos autores.

Para análise dos planos de disciplinas, utilizamos, como instrumento de auxílio, o software Interface de $R$ pour les Analyses Multidimensionnelles de Textes e de Questionnaires (IRaMuTeQ).

Realizamos a leitura dos 14 planos recebidos, momento em que selecionamos os conteúdos das ementas e organizamos, em um único documento de texto, inserindo-o no software, conforme orientação de seu tutorial (CAMARGO; JUSTO, 2013). Posteriormente configuramos o programa e foi gerada uma nuvem com as 15 palavras mais recorrentes.

No que se refere ao tratamento com as fontes, não nos interessou julgá-las, mas interrogá-las, entendendo-as como artefatos culturalmente construídos e repletos de intencionalidade (BLOCH, 2001). Ao explorarmos os documentos, analisamos as pistas e indícios (GINZBURG, 1989) deixados pelas fontes e as intencionalidades dos autores sobre o tema avaliação. 


\section{AVALIAÇÃO DO ENSINO E DA APRENDIZAGEM NAS DISCIPLINAS DA AMÉRICA LATINA}

Este estudo está fundamentado em 14 planos disponibilizados por 13 instituições de ensino da América Latina (ISEFFD/ARG, UM/ARG, UDEC/CHL, UDA/CHL, UNAB/CHL, UNACH/CHL, UCO/COL, CESMAG/COL, UNL/ECU, UABC/MEX, UDELAR/URY, UNELLEZ/VEN, UNEG/VEN). Como procedimento de análise das fontes, realizamos a leitura na íntegra desses documentos. Além disso, estabelecemos um cruzamento de dados entre a proposta das ementas com os objetivos e os conteúdos programáticos, obtendo como resultado as discussões centrais dessas disciplinas.

No intuito de darmos visibilidade ao que cada uma delas discute sobre avaliação do ensino e da aprendizagem, lançamos os conteúdos das ementas no IRaMuTeQ, obtendo, assim, a nuvem de palavras representada na Figura 1.

FIGURA 1 - Nuvem de palavras das ementas

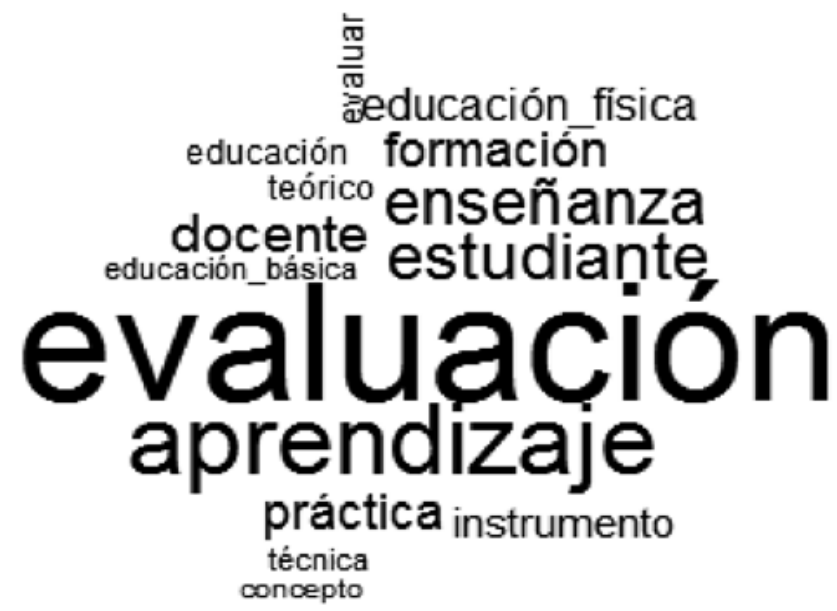

Fonte: Elaboração dos autores.

De acordo com a Figura 1, ressaltamos que as palavras mais recorrentes são também aquelas que correspondem aos objetivos e conteúdos trabalhados nas disciplinas. Nesse sentido, assumimos, como critério de análise, as discussões centrais envolvendo o tema avaliação do ensino e/ou 
aprendizagem em que seus conteúdos direcionam para ações avaliativas orientadas à prática do professor ou do aluno.

A recorrência à nuvem de palavras (Figura 1) evidencia a centralidade que se dá nas disciplinas quando se propõe discutir e ensinar a avaliação do processo de ensino e de aprendizagem. Nesse caso, temos articulação com a formação docente, suas implicações no contexto da educação básica, projetando possibilidades de práticas avaliativas em que se estabelece a relação teoria e prática. Esse movimento evidencia-se com a presença de palavras como "instrumento", "técnica”, "prática”, articulando-se também com a "teoria" e a "concepção de avaliação" que lhe oferecem suporte, relacionando-se com a "Educação Física" no contexto da educação profissional.

Estudar a avaliação, em específico o processo de ensino e de aprendizagem, significa pensá-la na própria formação, como também projetá-la para o contexto de atuação profissional, configurando uma perspectiva que the oferece fundamento para uma concepção formativa que está diretamente vinculada à compreensão de profissionalidade docente. Sendo assim, projeta-se que esse futuro professor atuará num determinado contexto com determinada disciplina escolar.

É importante considerar que as terminologias utilizadas demandam concepção que geram, por sua vez, uma derivação daquilo que será trabalhado no curso de formação de professores. Ela circunscreve aquilo que constitui o corpus de saberes selecionados para ser ensinado sobre avaliação na formação inicial de professores de Educação Física. Um indicador dessa configuração está na própria nomenclatura definida para a disciplina, ou seja, estudar uma avaliação "aplicada" que se utiliza de "estatística", pensa a "aprendizagem", traduz-se em "metodologias" na correlação com a "Educação Física", oferece pistas da necessidade de discutir o tema de maneira geral, projetando o modo como pode ser ela pensada e traduzida na prática do ato avaliativo.

Entretanto, estabelecer a correlação entre "currículo e avaliação" (UNAB/CHL), por exemplo, é definir que a disciplina dê conta de dois campos de discussões conceituais: os debates sobre currículo e sobre a avaliação. Isso evidencia 
uma configuração em que é necessário fragmentar o debate da avaliação com outros objetos de estudo da formação de professores. Nesse ponto, é preciso estabelecer uma diferenciação da disciplina ofertada no Equador, que tem a terminologia de "avaliação curricular" (UNL/ECU), pois essa nomenclatura nos oferece pistas do direcionamento do debate da avaliação do ensino e da aprendizagem para a avaliação institucional, configurando, assim, um campo de ensino e de estudo que amplia o próprio conceito que se tem sobre a temática.

É importante ainda destacar que, nos títulos das 14 disciplinas ofertadas, cinco apresentam uma discussão ampla da temática (avaliação educacional), indiciando abarcar a avaliação do ensino e da aprendizagem, do sistema e institucional; quatro direcionam o método e sua correlação com a área de conhecimento (Metodologia da Avaliação e Estatística Aplicada, Metodologia e Avaliação da Educação Física, Avaliação Aplicada e Avaliação da Atividade Física e Desporto); três especificamente a avaliação da aprendizagem; e duas o currículo.

Com intuito de evidenciarmos como essas discussões se apresentam, buscamos nos planos elementos que nos ajudassem a identificar o que é considerado central no ensino da avaliação. Desse modo, observamos que os conteúdos convergem em seis aspectos, conforme sinalizado na Tabela 1. 
TABELA 1 - Pontos de convergências entre os planos

\begin{tabular}{|c|c|c|}
\hline $\begin{array}{l}\text { PONTOS EM COMUM ENTRE OS PLANOS SOBRE } \\
\text { AVALIAÇÃO DO ENSINO E APRENDIZAGEM }\end{array}$ & INSTITUIÇÕES/UNIVERSIDADES & PORCENTAGEM \\
\hline Aspectos históricos, teóricos e legais da avaliação & $\begin{array}{l}\text { UDEC/CHL, UDA I/CHL, UDA II/CHL, UNAB/ } \\
\text { CHL, UNACH/CHL, UCO/COL, CESMAG/ } \\
\text { COL, UNL/ECU, UABC/MEX, UDELAR/URY, } \\
\text { UNELLEZ/VEN E UNEG/VEN }\end{array}$ & 85,7 \\
\hline Instrumentos avaliativos & $\begin{array}{l}\text { CESMAG/COL, UDELAR/URY, UABC/ } \\
\text { MEX, UNAB/CHL, UNL/ECU, UNEG/VEN, } \\
\text { UNACH/CHL, UDA II/CHL, UNELLEZ/ } \\
\text { VEN E UDEC/CHL }\end{array}$ & 71,4 \\
\hline $\begin{array}{l}\text { Fases/funções da avaliação: diagnóstica, formativa } \\
\text { e somativa }\end{array}$ & $\begin{array}{l}\text { UABC/MEX, UDA I/CHL, UCO/COL, } \\
\text { UDEC/CHL E UNELLEZ/VEN }\end{array}$ & 35,7 \\
\hline $\begin{array}{l}\text { Avaliação segundo seus agentes: autoevaluación, } \\
\text { coevaluación e heteroevaluación }\end{array}$ & $\begin{array}{l}\text { UNL/ECU, UNEG/VEN, UDA II/CHL, } \\
\text { UDEC/CHL e UNELLEZ/VEN }\end{array}$ & 35,7 \\
\hline $\begin{array}{l}\text { Avaliação na Educação Física: cualitativa e } \\
\text { cuantitativa }\end{array}$ & $\begin{array}{l}\text { UNEG/VEN, UDELAR/URY, CESMAG/ } \\
\text { COL e UNELLEZ/VEN }\end{array}$ & 28,5 \\
\hline Avaliação pautada nos aspectos físicos e motores & ISEFFD/ARG e UM/ARG & 14,28 \\
\hline
\end{tabular}

Fonte: Elaboração dos autores.

Observamos, conforme a Tabela 1, que 85,7\% dos planos apresentam, como parte introdutória, os "aspectos históricos, teóricos e legais da avaliação”. Em uma disciplina (UDEC/CHL), essa discussão é anunciada em sua ementa: “permitan al estudiante tener claridad sobre cómo ha evolucionado el concepto de evaluación y las implicancias que esto tiene [...]”. Em outras quatro, encontramos os objetivos: "Comprenderlos principales conceptos asociados a evaluación en educación” (Uda II/CHL); “Conocer la historia de la Evaluación Educacional distinguiendo sus momentos y oportunidades para la comprensión de su processo" (UNACH/CHL); "Informar sobre la reglamentación que regula la evaluación para que se tenga claridade" (UCO/COL); e "Interpretar referentes teóricos que le permiten establecer un fundamento conceptual sobre el origen, definición, objetivos y características de la evaluación” (CESMAG/COL). E, em sete planos, essa discussão é abordada nos seguintes conteúdos: "Conceptos generales y específicos de la evaluación en la Educación Física” (UDA I/CHL); "Conceptualización, principios y funciones de la evaluación" (UNAB/CHL); "La evaluación como campo de estudio" (UDELAR/URY); "Macro currículo y su importancia en el desarrollo de la evaluación” (UNEG/VEN); “Conceptos básicos 
de evaluación y de calidad" (UNL/ECU); "Bases teóricas de la evaluación" (UABC/MEX); "Fundamentos legales de la evaluación del aprendizaje” (UNELLEZ/VEN).

Apresentar os fundamentos históricos pode ser compreendido, tendo em vista que, desde o processo civilizatório, houve alguma forma de avaliação: ela nasceu com o próprio homem e, até se tornar o que conhecemos hoje por avaliação educacional, passou por diversas transformações, tornando-se uma atividade complexa, fundamentada nos pensamentos descritivo, analítico e crítico (VIANNA, 2000). Diante disso, é possível discutir, na formação, de que maneira ela foi assumindo características diferenciadas em sua trajetória histórica, concebida ora como ato de medida, ora como prática normativa e classificatória, com base em uma lógica somativa, passando a assumir aspectos qualitativos numa lógica formativa.

Casanova (1997, p. 18) salienta que é preciso esclarecer o caminho pelo qual a avaliação foi sendo desenvolvida, centrando-se em vertentes de reflexão que auxiliem na compreensão da situação atual e na superação das falhas que se arrastam ao longo do tempo. Para ela, isso ajudará a formular uma proposta válida de avaliação dos processos educativos que conjugue todas as exigências, requerendo sua aplicação rigorosa e objetiva. Para isso, a autora destaca, como eixos: a aparição da avaliação e sua aplicação no campo educativo; a evolução conceitual; a importância atual da avaliação nos sistemas educativos; e a avaliação no sistema educativo do país.

Sobre as terminologias utilizadas na avaliação, Lafourcada (1972) e Hoffmann (1991) ressaltam a importância de esclarecer as imprecisões conceituais presentes nos termos testar, medir e avaliar. Nessa mesma direção, Santos Guerra (1998, p. 13) expressa que a forma de entender a avaliação condiciona o processo de ensino e aprendizagem. Na visão do autor, a utilização da avaliação como instrumento de diagnóstico, de aprendizagem e de compreensão direcionada à melhoria tem menor presença que a avaliação como mecanismo de controle, de seleção, de comparação e de medição. Por isso, a importância de esclarecer esses aspectos com os futuros professores. 
Além disso, evidencia um deslocamento da ideia de avaliação como sinônimo de nota, na medida em que se configura em um ato humano que tem por objetivo e fundamento estabelecer juízo de valor sobre determinado sujeito, fato ou objeto. A entrada pela história permite que o aluno em formação amplie a própria compreensão do que é avaliar e de como se configura em diferentes contextos sociais, e não exclusivamente a escola.

$\mathrm{Na}$ escola, outros sentidos e entendimentos foram alcançados à proporção que foi servindo como balizador da determinação de uma hierarquização entre os sujeitos, os saberes ou na sua versão classificatória e certificadora, sendo usada para aprovar e reprovar; portanto, afastando-se do seu processo de acompanhamento dos processos formativos direcionados para a qualificação do ensino e da aprendizagem.

Já sobre os aspectos legais, Santos Guerra (2003) destaca que a avaliação é condicionada por disposições legais que a inspiram e regulamentam. Essas disposições são permeadas de uma filosofia que dá sentido à forma de praticar a avaliação. Além disso, unificam os momentos, a nomenclatura e seus conteúdos.

É importante considerar que o debate sobre a legislação se faz relevante quando observamos um movimento em que as determinações das avaliações externas (de sistemas ou larga escala) acabam configurando-se como delimitadoras do modo como a avaliação é entendida e trabalhada internamente no contexto da atuação profissional na escola. A identificação dos marcos legais e a maneira como eles têm direcionado uma determinada política de avaliação em cada país se fazem importantes para compreendermos como o cenário macro interfere na autonomia do trabalho pedagógico do professor e direciona esse fazer avaliativo para atender a essa política externamente orientada.

Desse modo, observamos nos planos que se pretende oferecer aos professores em formação as bases sobre os processos que influenciaram o campo; discutir as diferenças conceituais entre medida, qualificação e avaliação (UNELLEZ/VEN, UNACH/CHL, UNEG/VEN, UDA I/CHL e UDEC/CHL), para que adquiram o domínio conceitual sobre o tema; e apresentar seus 
6 UDEC - Sacristán e Pérez (1996), Santos Guerra (1996), Castillo (2002), Chile (2010); UDA II/CHL - Chile (2008), Monereo (2009), Hein e Taut (2010): UDA I/CHL - Blázquez Sánchez (1997), Monedero (1998); UNAB - Ahumada (1983), Casanova (1997) UNACH/CHL - Castillo e Diago (2003), Castro, Correa e Lira (2006); UCO/COL - Colômbia (1994); Casanova (1997) CESMAG/COL - Berton (1997), Monedero (1998); UNL/ECU - Ecuador (2010) Castillo e Diago (2012); UABC/ MEX - Blásquez Sánchez (1993), México (2002) UDELAR/URY - Berton (1997), Monedero (1998) UNELLLEZ/VEZ - Venezuela (1980, 2005); UNEG/VEN Bruzual e Sánchez (1998) Carli e Orighuela (2013). fundamentos legais (UCO/COL, UNELLEZ/VEN e UABC/MEX), para que conheçam o regulamento que rege a avaliação, as diretrizes e resoluções que a normatizam nos diferentes níveis educativos nacionais (Colômbia, Venezuela e México).

Para respaldarmos as questões relacionadas à fundamentação histórica e legal da avaliação, encontramos, na UDEC/CHL, quatro obras; na UDA II/CHL, três; e, na UDA I/CHL, UNAB/CHL, UNACH/CHL, UCO/COL, CESMAG/COL, UNL/ECU, UABC/MEX, UDELAR/URY, UNELLEZ/VEN e UNEG/VEN, duas. ${ }^{6}$ Ainda com base nas bibliografias, observamos que algumas obras são utilizadas em diferentes instituições e países, como o caso de Monedero (Chile, Colômbia e Uruguai), Casanova (Chile e Colômbia) e Bertoni (Colômbia e Uruguai).

$O$ segundo ponto mais expressivo entre as disciplinas (CESMAG/COL, UDELAR/URY, UABC/MEX, UNAB/CHL, UNL/ECU, UNEG/VEN, UNACH/CHL, UDA II/CHL, UNELLEZ/VEN e UDEC/CHL) refere-se aos "instrumentos avaliativos" (71,4\%). Em quatro instituições, essa discussão é sinalizada exclusivamente na unidade de conteúdos: "Técnicas e instrumentos de evaluación” (CESMAG/COL); “Construcción de instrumentos de evaluación en diferentes ámbitos de Educación Física” (UDELAR/URY); "Selección y elaboración de instrumentos de evaluación de los aprendizajes: instrumentos de evaluación: listas de cotejo y escalas de estimación, la rúbrica y las escalas de aprendizaje, tipos de pruebas: ensayos, objetivas y contextualizadas, tabla de especificación para elaboración de pruebas" (UNEG/VEN) e; "¿Como evaluar?: Uso de técnicas, instrumentos y recursos" (UNELLEZ/VEN).

Outras seis instituições apresentam a temática tanto na unidade de conteúdos quanto nos objetivos da disciplina: "Diseñar y aplicar instrumentos de evaluación de la enseñanza de la educación física” (UABC/MEX); "Conocer y aplicar los criterios técnicos para la construcción de procedimientos, los propósitos de la evaluación y los instrumentos evaluativos presentes en el proceso enseñanza aprendizaje" (UNAB/CHL); "Establecer criterios y elaborar instrumentos para evaluar resultados de aprendizaje en Educación Física, Deportes y Recreación” (UNL/ECU); “Planificar procesos de evaluación de 
los aprendizajes, construyendo los instrumentos de evaluación correspondientes" (UNACH/CHL); "Conocer y comprender distintos tipos de evaluación: sus situaciones de aplicación, procedimientos $\mathrm{y} / \mathrm{o}$ instrumentos. Comprender cómo evaluar distintos tipos de contenidos curriculares. Conocer los fundamentos teóricos y técnicos de los instrumentos evaluativos y aplicarlos en su construcción” (UDA II/CHL); e "Reconocer componentes, finalidad y técnicas para el diseño de instrumentos de evaluación [...] instrumentos de informes: cuestionario, informes, guía de ejercicios, bitácora de aprendizaje, portafolio de aprendizaje, contrato de aprendizaje" (UDEC/CHL).

Pela grande presença nos planos $(71,4 \%)$, observamos a relevância dessa temática nas disciplinas e no curso de formação de professores. Sobre os instrumentos avaliativos, Castillo e Diago (2012, p. 308) salientam que um bom profissional é aquele que sabe escolher, em cada circunstância, os instrumentos que melhor se adaptem à situação, não podendo limitar os testes e exames tradicionais como únicas ferramentas para avaliar os alunos. A variação dos tipos (observação, entrevistas, pesquisas), assim como as diferentes formas de expressão (oral, escritas, gráficas), devem ser utilizadas à medida que proporcionem ao professor um conhecimento contínuo e adequado ao progresso do estudante.

No entanto, discutir os instrumentos nos cursos de formação de professores está além de apresentá-los aos alunos e estabelecer variações: é preciso também atribuir sentido ao processo avaliativo e à perspectiva epistemológica que lhe oferecem suporte, pois, caso haja debate sobre "como fazer a avaliação", sem articulação com as problemáticas da escola, isso contribuirá para que os professores, ao ingressarem na educação básica, produzam registros avaliativos diversificados, sem, contudo, compreender os sentidos da avaliação (HOFFMANN, 2000).

Assim, Hoffmann (2005) e Santos, Maximiano e Frossard (2016) pontuam que não há instrumentos avaliativos descontextualizados das perspectivas pedagógicas que as fundamentam. Ou seja, não é o instrumento que determina sua concepção, mas a concepção que lhe oferece suporte 
e orienta a intencionalidade do avaliador. Essa preocupação pode ser evidenciada principalmente nos objetivos das disciplinas da UDA II/CHL e UDEC/CHL: “Conocer y comprenderdistintos tipos de [...] instrumentos. Conocer los fundamentos teóricos y técnicos de los instrumentos evaluativos [...]"; "Reconocer componentes, finalidad y técnicas para el diseño de instrumentos".

Ainda relacionado aos instrumentos e ao curso de formação de professores, Santos et al. (2018), ao analisarem o papel da formação inicial em Educação Física na constituição de um corpus de saberes teóricos e práticos que fundamenta o seu ensino em sete universidades federais brasileiras, sinalizam o distanciamento entre as experiências avaliativas e a atuação docente, acenando, assim, com a necessidade de os cursos de formação de professores em Educação Física assumirem, como eixo central, a prática de ensino.

É preciso, ainda, discutir e criar instrumentos avaliativos que reconheçam a especificidade do saber que ensina a Educação Física (SANTOS, 2005, 2008). Quando observamos essa questão relacionando com o modo como o debate se apresenta nos currículos prescritos, identificamos que três disciplinas (UDELAR/URY, UABC/MEX, UNL/ECU) estabelecem essa preocupação de pensar na definição de conteúdos e objetivos que direcionam o debate para a Educação Física. Ou seja, as concepções avaliativas são comuns a todas as áreas de conhecimento, mas o saber privilegiado por essas áreas é específico, levando em consideração a natureza daquilo que se propõe a ensinar.

Nesse caso, estudos como os de Santos et al. (2015, 2016a) têm sinalizado que, na Educação Física, tem sido privilegiado o "saber com" em vez do "falar de" e "escrever sobre". No entanto, o fato de privilegiar tal dimensão não exclui a possibilidade de materializar o saber incorporado pelo domínio de uma atividade e o produzido pela dimensão relacional em dispositivos de leitura, pois a articulação entre essas diferentes formas de avaliar demonstra que é possível abranger a complexidade dos conhecimentos ensinados e aprendidos na Educação Física, assim como a relação que os alunos estabelecem com os saberes específicos desse componente curricular. 
Pensar a avaliação do ensino e da aprendizagem na Educação Física, tendo como centralidade o esporte, a recreação, o lazer, conduz para o que se ensina e o que se aprende, bem como o que se valoriza na ação pedagógica do professor. Isso implica a necessidade de pensar especificamente a construção de instrumentos que deem visibilidade ao modo como os alunos se apropriam desse saber que se constitui pelo domínio das práticas corporais, sua reflexão e pela produção do conhecimento derivado dela.

Outro aspecto em comum refere-se às "fases/funções da avaliação diagnóstica, formativa e somativa” (35,7\%) presentes em planos de cinco instituições (UABC/MEX, UDA I/CHL, UCO/COL, UDEC/CHL e UNELLEZ/VEN). Na UABC/MEX e UDEC/CHL, respectivamente, essa discussão apresenta-se tanto na unidade de conteúdo quanto no objetivo específico dessa unidade: "Identificar las fases de evaluación en la actividad física, mediante la descripción de su finalidad y características, para aplicarlas en el transcurso de un proceso educativo"; "Planificar de forma pertinente el proceso evaluativo, en relación a la finalidad: diagnóstica, formativa y sumativa”. Já a UDA I/CHL, UCO/COL e UNELEZ apresentam essa discussão na unidade de conteúdos: "Momientos evaluativos" (UDA I/CHL); "Fases del proceso de evaluación" (UCO/COL); e "Tipos de la evaluación del aprendizaje: diagnóstica, formativa y final o sumativa” (UNELLEZ/VEN).

Segundo Rabelo (1998), na avaliação diagnóstica, faz-se um prognóstico sobre as capacidades do aluno em relação a um novo conteúdo a ser abordado. É o momento de detectar dificuldades dos alunos para que o professor melhor conceba estratégias de ação para solucioná-las. Esse processo não tem finalidade probatória, assim como a avaliação formativa, cujo intuito é proporcionar informações acerca do desenvolvimento de um processo de ensino e aprendizagem tanto para o professor quanto para o aluno. Já a avaliação somativa normalmente é pontual, acontece no fim do processo de ensino, propõe um balanço somatório de uma ou várias sequências do trabalho, cuja principal função é dar certificado, titular.

Nessa mesma direção, Casanova (1997) salienta que, referentemente à temporalização, a avaliação inicial ou 
diagnóstica ocorre quando um aluno, pela primeira vez, chega à escola ou quando se começa uma nova unidade didática; de qualquer forma, é uma maneira de detectar o estágio em que se encontra o estudante. Sobre a processual ou formativa, destaca que consiste na avaliação contínua da aprendizagem do aluno e do ensino do professor mediante a recolha sistemática de dados, análises deles e tomada de decisões. Quanto ao tempo de duração desse processo, a autora salienta que esse é marcado pelos objetivos assinalados. Por fim, a avaliação final, como o próprio nome apresenta, realiza-se ao terminar um processo de ensino e aprendizagem. É uma avaliação que comprova os resultados obtidos, mas é necessário advertir que, por isso, não deve ter funcionalidade exclusivamente somativa.

É interessante observar que a definição das fases e funções da avaliação apresentada na Tabela 1 revela a preocupação em orientar a planificação e organização do processo avaliativo em um tempo determinado, já que ela vai constituir todos os tipos que correspondem à avaliação e são usados em momentos diferentes. Portanto ela sinaliza a necessidade de assumir a avaliação como direcionadora do trabalho pedagógico, na medida em que é assumida com sua função diagnóstica, oferecendo elementos para possibilitar a orientação do que será trabalhado ao longo do processo, traduzida na construção do plano de trabalho e na definição de objetivos, conteúdos e procedimentos metodológicos a serem adotados.

Posteriormente, fundamentados nessa planificação, avalia-se o processo com objetivo de reorientar e adequar, assumindo a avaliação na sua perspectiva formativa. E, no final, para constituir um produto que está diretamente relacionado com o que se delimitou e acompanhou do processo, ocorre a avaliação somativa, com objetivo de certificar, classificar, determinar. Observa-se, com isso, a preocupação das instituições que trabalham com essa discussão: pensar a avaliação como o eixo central da orientação, acompanhamento e consolidação do processo pedagógico.

O quarto aspecto identificado nas disciplinas refere-se aos "agentes da avaliação", definidos de acordo com as pessoas que a realizam, originando os processos de autoevaluación, 
coevaluación e heteroevaluación. Encontramos essa temática em 35,7\% dos planos, a saber: UNL/ECU, UNEG/VEN, UDA II/CHL, UDEC/CHL e UNELLEZ/VEN. Em todos se apresenta na unidade de conteúdo: "Marco curricular de la autoevaluación, la heteroevaluación y la coevaluación" (UNL/ECU); “Autoevaluación y coevaluación” (UNEG/VEN); "Distintos tipos de evaluación que apoyan el aprendizaje e involucran a los aprendices desarrollando la meta cognición: la autoevaluación y coevaluación” (UDA II/CHL); "Planificar de forma pertinente el proceso evaluativo, en relación a la finalidad: diagnóstica, formativa y sumativa; según agente: autoevaluación, heteroevaluación, coevaluación" (UDEC/CHL); e "Formas de participación en la evaluación del aprendizaje: autoevaluación, coevaluación y heteroevaluación" (UNELLEZ/VEN).

López-Pastor e Pérez Pueyo (2017) nos ajudam a compreender esses distintos tipos de avaliação, ao definirem que a autoevaluación é aquela que uma pessoa realiza sobre si mesma ou sobre o processo e/ou resultado pessoal. Na profissão docente, esse termo é geralmente utilizado para referir-se à autoavaliação dos alunos, mas também pode ser encontrado na referência à autoavaliação do professor, normalmente ligado aos processos de melhoria profissional. Na prática profissional, a autoevaluación deve ser introduzida de modo habitual entre os alunos e alunas, com diferentes graus de complexidade, de acordo com as idades, para que os estudantes sejam capazes de avaliar o próprio trabalho e o grau de satisfação que produz. Só é preciso dar-lhes orientações dos aspectos a serem autoavaliados, para que os realizem com seriedade, e não arbitrariamente (CASANOVA, 1997, p. 86).

Em contrapartida, a coevaluación refere-se à avaliação entre pares: consiste na avaliação conjunta de uma atividade ou trabalho específico. Nesse caso, após a prática de uma série de atividades ou no final de uma unidade didática, estudantes e professor, ou professores, podem avaliar certos aspectos que julgam interessante destacar. Ao contrário da coevaluación, a heteroevaluación refere-se aos processos de avaliação realizados por pessoas que pertencem a diferentes níveis, ou seja, não 
cumprem a mesma função, como familiares, professores que ensinam outras disciplinas, agentes externos.

A opção em ensinar os agentes responsáveis pelo processo avaliativo na formação de professores de Educação Física sinaliza a preocupação em evidenciar que essa temática não está centralizada apenas no professor, tampouco no aluno; ela envolve diferentes sujeitos que têm responsabilidades específicas, considerando aquilo que é da sua governabilidade. Simultaneamente, traz uma delimitação de diferenciação conceitual, tanto evidenciando o objetivo de direcionar a melhoria da qualidade dos processos de aprendizagem na autoavaliação do aluno quanto adequando os processos de ensino e a melhoria da prática pedagógica do professor. Essa temática amplia, assim, a responsabilidade da avaliação para aluno, professor e agentes externos, influenciando na formação de todos os envolvidos no processo.

A discussão da avaliação nas dimensões cualitativa e cuantitativa está presente em 28,5\% dos planos (UNEG/VEN, UDELAR/URY, CESMAG/COL e UNELLEZ/VEN). A UDELAR/URY e a CESMAG/COL abordam essa temática na unidade de conteúdo e nos objetivos: "Presentar la doble dimensionalidad de la evaluación del aprendizaje en Educación Física (cualitativa y cuantitativa), sus especificidades y sus posibles abordajes en el campo educativo” (UDELAR/URY); “Compara a partir de referentes teóricos los fundamentos conceptuales establecidos para cada paradigma generando para sí, una perspectiva clara sobre su razón de ser y el objeto de su establecimiento en los contextos educativos y sociales" (CESMAG/COL). Já na UNEG/VEN e na UNELLEZ/VEN, encontramos essa discussão na unidade de conteúdos: "Modalidades de la evaluación: Cuantitativa y Cualitativa” (UNEG/VEN); “Tendencia de la evaluación del aprendizaje: enfoques y modelos de evaluación" (UNELLEZ/VEN).

Sobre as dimensões qualitativa e quantitativa, especificamente na Educação Física, Sarni (2006) afirma que ambas circulam no nível educativo. No que se refere à dimensão qualitativa, a intenção é avaliar para a compreensão do que foi aprendido pelo aluno, num sentido contínuo e formativo. Tem como vantagem a melhoria da aprendizagem, propiciando 
aos estudantes autonomia e autorregulação dos processos. Isso permite ao aluno maior participação e envolvimento na avaliação. Nesse aspecto, a UDELAR/URY utiliza autores como Camilloni (1998), Sales (2003), Hernandez et al. (2004), López-Pastor e Pérez Brunicardi (2004). A CESMAG/COL referencia para esse tópico a obra de Flórez Ochoa (2002). Na UNELLEZ/ VEN, encontramos obras de Alves e Acevedo (1999) e Shaw (2003). Já na UNEG/VEN, identificamos, para a avaliação qualitativa, Bruzual e Sánchez (1998) e Alves e Acevedo (2010).

A dimensão quantitativa refere-se à avaliação do aluno com parâmetros observáveis e quantificáveis (centímetros, peso, tempo). Realiza-se para testar certas capacidades medíveis e situar o avaliado, tanto em relação aos outros como a si mesmo, na própria evolução de tempo ou devido a escalas preestabelecidas, e é utilizada para medir o rendimento físico, motriz ou desportivo. A UDELAR/URY propõe discutir a avaliação com enfoque quantitativo, abordando os testes na Educação Física e a cineantropometria. Relaciona essa discussão às seguintes bibliografias: Litwin e Fernández (1977), Pila Teleña (1985), Hahn (1988), Weineck (1988), Molnar (1996), Blázquez Sánchez (1997) e Molnar, Dávila e Kohan (2000). A CESMAG/COL utiliza para esse tópico a obra de Flórez Ochoa (2002). Já na UNEG/VEN e UNELLEZ/VEN, não são apresentadas bibliografias que se centralizam nessa temática.

É importante destacar que, nos documentos, não há, na sinalização do trabalho com avaliação qualitativa e quantitativa, um processo de contraposição de um com o outro, mas o princípio de complementação. Portanto não se percebe nos planos a defesa de uma dimensão devido às críticas à outra, mas a necessidade de o futuro docente ter, no processo formativo, a compreensão de que, do ponto de vista tanto dos métodos quantitativos quanto dos qualitativos, ambos podem contribuir para o acompanhamento do aprendizado dos alunos.

Entretanto, pela dimensão quantitativa, consegue-se perceber aspectos motores, físicos, por meio de unidade de medida que se fundamenta na cineantropometria, o que evidencia também uma aproximação com uma concepção de Educação Física voltada para seus aspectos anatomofisiológico, tendo 
por objetivo produzir indicadores estatísticos que permitem o acompanhamento do desenvolvimento de algumas capacidades físicas, habilidades motoras, por meio de protocolo de testes predefinidos. Esse movimento se reforça quando analisamos a avaliação do ensino e da aprendizagem, apresentando em comum a discussão dos aspectos físicos e motores.

Ainda relativamente à avaliação do ensino e da aprendizagem, duas instituições (UM/ARG e ISEFFD/ARG) apresentam, em comum, a discussão pautada nos "aspectos físicos e motores", representando $14,28 \%$. Ao cruzarmos os dados das ementas, objetivos e conteúdos dessas disciplinas, encontramos a centralidade dessa discussão em palavras, tais como evaluación, técnica, aprendizaje, instrumento, estudientes, formación, práctica, enseñanza, evaluar e Educación Física, presentes na Figura 1. Especificamente, destacam, em suas ementas:

Los potenciales beneficios causados por la adecuada utilización del entrenamiento físico son innegables en el ámbito del deporte y del rendimiento motor. También son fundamentales en la vida del estudiante para incorporar hábitos de vida saludable como prevención y promoción de la salud. (UM/ARG)

Preguntas tales como ¿con quién estoy trabajando? ¿qué herramientas puedo usar para identificar el verdadero nivel del alumno / entrenado y de su grupo de pertenencia? obligan a hacer un diagnóstico objetivo, confiable y útil para la posterior intervención que se aplicará. Eso es evaluar; juzgar la situación eficientemente, respetando las características del grupo a cargo. (ISEFFD/ARG)

Percebemos que ambas as disciplinas fornecem indícios da concepção de Educação Física presente nessas duas instituições da Argentina. Crisorio et al. (2015), ao analisarem o perfil profissional da área nesse país, evidenciam que, historicamente, a Educação Física tem se fundamentado nas ciências biomédicas e no desporto, buscando, no estado moderno, atender à educação física, moral e intelectual do cidadão. Atualmente, o caráter biológico e desportivo ainda baliza o campo, influenciando a formação de professores 
que atuam diretamente no contexto escolar e aqueles que se dedicam às pesquisas na área.

[...] atraviesa la formación tanto en los institutos de nivel terciario, como en las universidades [...]. Hemos observado una alta carga horaria de asignaturas de corte biológico (como anatomía, fisiología), de corte psico-biológico (psicologías evolutivas, psicología del aprendizaje, psicopedagogía, pedagogía o incluso las didácticas ligadas a criterios evolutivos o del desarrollo) que ligan de un modo u otro la formación de los futuros enseñantes a una matriz cuya productividad discursiva sobre la enseñanza se asienta en criterios 'científicos' de base orgánica. (CRISORIO et al., 2015, p. 32)

Isso se revela nos conteúdos programáticos das disciplinas, ao focalizarem "test, medición y evaluación, potencia y capacidade aeróbica, estadística aplicada, cineantropometria, cuantificación de la actividad física" (ISEFFD/ARG) e evaluación antropométrica, fuerza muscular, medición y capacidade anaeróbicas, campos de aplicación de las evaluaciones fisiológicas fuera del deportivo (UM/ARG).

Esses conteúdos são sustentados por bibliografias que tematizam testes e provas físicas (GEORGE; GARTH FISHER; VEHRS, 1996) e a mensuração (FLOOD, 1996; KIRKENDALL; GRUBER; JOHNSON, 1987; MORROW et al., 1995) na ISEFFD/ARG. Já na disciplina da UM/ARG, o foco das bibliografias indicadas são as medidas antropométricas (ESPARZA ROS, 1993; GRIS et al., 2004; MARFELL-JONES et al., 2001), as bases fisiológicas (BOSCO, 2000; MC ARDLE; KATCH; KATCH, 1996) e treinamento desportivo (KUZNETSOV, 1989; PLATONOV, 1993; VALDIVIELSO, 2004).

Identificamos que a concepção de Educação Física pautada no caráter biológico e desportivo fundamenta os cursos e, consequentemente, as disciplinas de avaliação. Ao analisarmos as bibliografias desses planos, vemos que, de modo geral, elas centram suas práticas privilegiando as aprendizagens motoras, sob o enfoque desportivo ou aspectos fisiológicos, priorizando as medidas antropométricas. 


\section{CONSIDERAÇÕES FINAIS}

Ao objetivarmos, neste estudo, compreender como a avaliação do ensino e da aprendizagem é prescrita nas disciplinas específicas sobre o tema nos cursos de formação de professores em Educação Física nos países da América Latina e utilizar o IRaMuTeQ como ferramenta de auxílio, identificamos que, de maneira geral, a centralidade nas 14 disciplinas está em discutir e ensinar a avaliação considerando suas implicações para o contexto da educação básica, as possibilidades de práticas avaliativas e a necessidade da relação teoria e prática. Esse movimento evidencia-se com a presença de algumas palavras, como "instrumento", "técnica”, "prática”, articulando-se também com a "teoria” e a "concepção de avaliação" que lhe oferece suporte, relacionando-se com a "Educação Física” no contexto da educação profissional.

O software (IRaMuTeQ) mostrou-se uma ferramenta importante para a realização deste estudo, na medida em que nos possibilitou um olhar criterioso sobre as fontes, qualificando o processo de categorização e, consequentemente, dos resultados, e potencializando a pesquisa.

Sobre o que discutem as disciplinas, com base nos seus títulos, observamos que: cinco apresentam uma discussão ampla da temática (avaliação educacional); quatro direcionam o método e sua correlação com a área de conhecimento; três anunciam especificamente a avaliação da aprendizagem; e duas o currículo. Em todas há, ao menos, uma unidade para discutir a avaliação do ensino e aprendizagem, porém notamos a ausência das outras modalidades da avaliação.

É preciso considerar que ensino e aprendizagem não são sinônimos e que as delimitações de suas ações avaliativas direcionam as análises para a prática do professor ou do aluno. Sendo assim, quando nos referimos à avaliação do ensino, consideramos que o objetivo é oferecer informações que permitam ao professor orientar sua prática pedagógica, direcionando-a aos processos de aprendizagem dos alunos. Já a avaliação das aprendizagens é aquela focada nos alunos, cujo objetivo é potencializar seus processos formativos, contribuindo para sua autorregulação. 
Faz-se necessário, ainda, ampliarmos as análises, em estudos futuros, sobre como as nomenclaturas das disciplinas direcionam as delimitações dos temas e conteúdos a serem ensinados. Ou seja, se, no título da disciplina, são indicadas palavras como avaliação, currículo e didática, há indícios de que se tem o agrupamento de três disciplinas em uma, reduzindo o próprio ensino da avaliação.

Ao investigarmos o que é considerado relevante a ser trabalhado sobre o ensino da avaliação, observamos que os conteúdos convergem sobre seis aspectos comuns entre os planos: aspectos históricos, teóricos e legais; instrumentos avaliativos; fases/funções (diagnóstica, formativa e somativa); agentes avaliativos (autoavaliação, coavaliação e heteroavaliação); relacionados à Educação Física (qualitativa e quantitativa); e pautados nos aspectos físicos e motores.

Nesse sentido, pretende-se oferecer aos professores em formação as bases históricas sobre os processos que influenciaram o campo; discutir as diferenças entre medida, qualificação e avaliação, para que adquiram um domínio conceitual sobre o tema no campo geral e na Educação Física; apresentar seus fundamentos legais para que conheçam o regulamento que rege a avaliação, as diretrizes e resoluções que a normatizam nos diferentes níveis educativos nacionais e discutir e criar instrumentos que auxiliem no processo avaliativo.

Apesar de se tratar de cursos de formação de professores de uma área específica (Educação Física), identificamos que, das 14 disciplinas analisadas, seis (ISEFFD/ARG, UM/ARG, UDELAR/URY, UABC/MEX, UNL/ECU e UDA I/CHL) fazem relação direta com a área.

Compreendemos que estudar a avalição nos seus diferentes aspectos, conforme apresentado ao longo desse estudo, ajuda a pensar suas implicações nos cursos de formação de professores, tendo em vista sua projeção para os processos de ensino e de aprendizagem no futuro contexto de atuação profissional.

No que se refere às disciplinas aqui estudadas, é preciso considerar que as terminologias utilizadas em seus títulos traduzem uma determinada concepção de avaliação e de 
Educação Física; essas, por sua vez, têm gerado derivações daquilo que será trabalhado no curso e projetado para o futuro profissional. Assim, elas representam a centralidade daquilo que é ensinado sobre avaliação na formação inicial de professores de Educação Física em sete países da América Latina, indicando a necessidade de discutir o tema de maneira geral, projetando o modo como ela pode ser pensada e traduzida na prática do fazer avaliativo do professor. Além disso, foi possível perceber que o debate do tema nas 14 disciplinas avança para apresentar os diferentes aspectos que envolvem a avaliação educacional, porém carece de aprofundamentos sobre as especificidades do avaliar na disciplina de Educação Física escolar.

Com base nos achados deste estudo, sinalizamos a necessidade de pesquisas que investiguem como a avaliação institucional e de sistemas é discutida nas disciplinas específicas de avaliação nos cursos de formação de professores em Educação Física nos países da América Latina.

\section{REFERÊNCIAS}

AHUMADA, P. Principios y procedimientos de evaluación educacional. Valparaíso: Universitaria UC, 1983.

ALVES, E.; ACEVEDO, R. La evaluación cualitativa: reflexión para la transformación de la realidad educativa. Valência: Cerined, 1999.

ALVES, E.; ACEVEDO, R. Evaluación cualitativa: orientaciones para la práctica en el aula. Valência: Cerined, 2010.

BATISTA, M. A. M. Avaliação: um intervir sobre a população do conhecimento. Pensar a Prática, Goiânia, v. 3, p. 65-71, jul./jun. 2000.

BERTONI, A. et al. Evaluación: nuevos significados para una práctica compleja. Bogotá: Kapelusz, 1997. (Colección triângulos pedagógicos).

BLÁZQUEZ SÁNCHEZ, D. Perspectivas de la evaluación en educación física y deporte. Apuntes: Educación Física I Esports, Barcelona, n. 31, p. 5-16, ene./mar. 1993.

BLÁZQUEZ SÁNCHEZ, D. Evaluar en Educación Física. Barcelona: INDE, 1997.

BLOCH, M. L. B. Apologia da história ou o ofício do historiador. Rio de Janeiro: Jorge Zahar, 2001.

BOSCO, C. La fuerza muscular. Barcelona: INDE, 2000. 
BRUZUAL, T.; SÁNCHEZ, J. Aproximaciones teóricas para una evaluación cualitativa. In: JORNADAS NACIONALES DE INVESTIGACIÓN HUMANÍSTICA Y EDUCATIVA, 4., 1998, Valência. Anais... Valência: Universidad de Carabobo, 1998.

CAMARGO, B. V.; JUSTO, A. M. IRaMuTeQ: um software gratuito para análise de dados textuais. Temas em Psicologia, Ribeirão Preto, SP, v. 21, n. 2, p. 513-518, maio 2013.

CAMILLONI, A. et al. La evaluación de los aprendizajes en el debate didáctico contemporáneo. Buenos Aires: Paidos, 1998.

CARLI, M.; ORIGHUELA, N. La evaluación educativa. México: La Muralla, 2013.

CASANOVA, M. A. Manual de la evaluación educativa. Madrid: La Muralla, 1997.

CASTILLO, S. Evaluación educativa y promoción escolar. Madrid: Pearson, 2002.

CASTILLO, S.; DIAGO, J. C. Evaluación y promoción escolar. Madrid: Pearson, 2003.

CASTILLO, S.; DIAGO, J. C. Evaluación educativa de aprendizajes y competencias. Madrid: Pearson, 2012.

CASTRO, F.; CORREA, M.; LIRA, H. Curriculum y evaluación educacional. Concepción: Universidad del Bío, 2006.

CHILE. Ministerio de Educación. Evaluación para el aprendizaje. Santiago: Ministerio de Educación, 2008. Disponível em: <http://www.cpeip.cl/usuarios/ cpeip/File/2013materialespsp/1cicloepa.pdf $>$. Acesso em: 7 fev. 2018.

CHILE. Ministerio de Educación. Unidad de curriculum y evaluación. Marco y bases curriculares, programas de estudio y mapas de progreso correspondiente a la educación básica. Santiago: Ministerio de Educación, 2010. Disponível em: <www.mineduc.cl>. Acesso em: 6 fev. 2018.

COLÔMBIA. Ley n. 115, de febrero 8 de 1994. Ley general de educación. Santafé de Bogotá, 1994. Disponível em: <https://www.mineducacion.gov.co/1621/ articles-85906_archivo_pdf.pdf>. Acesso em: 8 jan. 2018.

CRISORIO, R. et al. Acerca de la formación en Educación Física en la República Argentina. In: SILVA, A. M.; BEDOYA, V. M. (Org.). Formação profissional em Educação Física na América Latina: encontros, diversidades e desafios. Jundiaí, SP: Paco Editorial, 2015.

EQUADOR. Ministerio de Educación. Ley orgánica de educación intercultural. Quito: Ministerio de Educación, 2010. Disponível em: <www.ducación.gob. ec>. Acesso em: 16 jan. 2018.

ESPARZA ROS, F. Manual de cineantropometría. Madrid: Femede, 1993.

FLOOD, D. K. Practical math for health fitness professionals. Champaign, IL: Human Kinetics, 1996.

FLÓREZ OCHOA, R. Evaluación pedagógica y cognición. Bogotá: Mc Graw Hill, 2002.

FREITAS, L. C. Avaliação educacional: caminhando pela contramão. 6. ed. Petrópolis, RJ: Vozes, 2014. 
FROSSARD, M. L. Avaliação educacional em educação física: um mapa da produção acadêmica de 1930-2014. 2015. 122 f. Dissertação (Mestrado em Educação Física) - Programa de Pós-Graduação em Educação Física, Universidade Federal do Espírito Santo, Vitória, 2015.

GALLARDO-FUENTES, F.; LÓPEZ-PASTOR, V. M.; CARTER-THUILLIER, B. ¿Hay evaluación formativa y compartida en la formación inicial del profesorado en Chile? Percepción de alumnado, professorado y egresados de una universidad. Psychology, Society, \& Education, Almería, v. 9, n. 2, p. 227-238, jul. 2017.

GEORGE, J. D.; GARTH FISHER, A.; VEHRS, P. R. Test y pruebas físicas. Barcelona: Paidotribo, 1996.

GINZBURG, C. Mitos, emblemas, sinais: morfologia e história. São Paulo: Companhia das Letras, 1989.

GRIS, G. M. et al. Estudio somatotípico en la población activa de Argentina. Apunts Medicina de l’Esport, Catalunya, v. 39, n. 144, p. 35-40, ago. 2004.

HAHN, E. Entrenamiento con niños. Barcelona: Martínez Roca, 1988.

HEIN, A.; TAUT, S. El uso de información evaluativa externa con fines formativos en establecimientos educacionales chilenos. Revista Iberoamericana de Evaluación Educativa, Madrid, v. 3, n. 2, p. 160-181, mayo 2010.

HERNANDEZ, J. L. et al. La evaluación en educación física: investigación y práctica en el ámbito escolar. Madrid: Graó, 2004.

HOFFMANN, J. Evaluación, mito y desafío. Educación y realidad. Porto Alegre: Mediação, 1991.

HOFFMANN, J. Avaliação mediadora: uma relação dialógica na construção do conhecimento. Porto Alegre: Mediação, 2000.

HOFFMANN, J. O jogo do contrário em avaliação. Porto Alegre: Mediação, 2005.

KIRKENDALL, D.; GRUBER, J.; JOHNSON, E. Measurements and evaluation for physical educators. Champaign, IL: Human Kinetics,1987.

KUZNETSOV, V. V. Metodología del entrenamiento de la fuerza para deportistas de alto nivel. Buenos Aires: Stadium, 1989.

LAFOURCADA, P. Evaluación de los aprendizajes. Bogotá: CINCEL, 1972.

LITWIN, J.; FERNÁNDEZ, G. Medidas, evaluación y estadísticas aplicadas a la educación física y el deporte. Buenos Aires: Stadium, 1977.

LÓPEZ-PASTOR, V.; PÉREZ BRUNICARDI, D. Análisis y debates sobre la evaluación en educación física. Novedades educativas, Buenos Aires, v. 16, n. 157 , p. 24-28, ene. 2004.

LÓPEZ-PASTOR, V. M.; PÉREZ PUEYO, A. (Coord.). Evaluación formativa y compartida en educación: experiencias de éxito en todas las etapas educativas. León: Universidad de León, 2017.

MARFELL-JONES, M. et al. Manual ISAK: estándares de medidas antropométricas internacionales. Durban: Sociedad Internacional para el Avance de la Cineantropometría, 2001. 
MC ARDLE, W. D.; KATCH, F. I.; KATCH, V. L. Fisiología del ejercicio: energía, nutrición y rendimiento humano. Madrid: Williams \& Wilkins, 1996.

MENDES, E. H.; NASCIMENTO, J. V. do; MENDES, J. C. Metamorfoses na avaliação em educação física: da formação inicial à prática pedagógica escolar. Movimento, Porto Alegre, v. 13, p. 13-37, jan./abr. 2007.

MÉXICO. Secretaria de Educación Pública. Plan de Estudios. Licenciatura en Educación Física. La educación física en las escuelas de educación básica (preescolar, primaria y secundaria). México: Secretaria de Educación Pública, 2002.

MOLNAR, G. Entrenamiento y deporte infantil. Montevidéu: Rosgal, 1996.

MOLNAR, G.; DÁVILA, J. L.; KOHAN, A. Control y evaluación del entrenamiento: repartido del diploma de preparador físico. Montevidéu: Rosgal, 2000.

MONEDERO, J. Bases teóricas de la evaluación educativa. Granada: Aljibe, 1998.

MONEREO, C. (Coord.). Pisa como excusa: repensar la evaluación para cambiar la enseñanza. Barcelona: Graó, 2009.

MORROW, J. R. et al. Measurement and evaluation in human performance. Champaign, IL: Human Kinetics,1995.

PILA TELEÑA, A. Evaluación de la educación física y los deportes: los test de laboratorio al campo. Madrid: Augusto E. Pila, 1985.

PLATONOV, V. N. El entrenamiento deportivo. Madrid: Paidotribo, 1993.

RABELO, E. H. Avaliação: novos tempos, novas práticas. Petrópolis, RJ: Vozes, 1998.

SACRISTÁN, J. G. 0 currículo: uma reflexão sobre a prática. Porto Alegre:

Artmed, 2000.

SACRISTÁN, J. G.; PÉREZ, A. Comprender y transformar la enseñanza. Madrid: Morata, 1996.

SALES, M. T. La evaluación de los aprendizajes desde una perspectiva de la complejidad. Folleto del curso ofrecido por el centro de Educación Física de la ISEF, Montevideo, 2003.

SANTOS GUERRA, M. Evaluación educativa 1: un proceso de diálogo, comprensión y mejora. 2. ed. Buenos Aires: Magisterio del Río de la Plata, 1996.

SANTOS GUERRA, M. Evaluar es comprender. Buenos Aires: Magisterio del Río de la Plata, 1998.

SANTOS GUERRA, M. Dime cómo evalúas y te diré qué tipo de professional y de persona eres. Revista Enfoques Educacionales, Santiago, v. 5, n. 1, p. 69-80, 2003.

SANTOS, W. dos. Currículo e avaliação na educação física: do mergulho à intervenção. Vitória, ES: Proteoria, 2005.

SANTOS, W. dos. Currículo e avaliação na Educação Física: práticas e saberes. In: SCHNEIDER, O. et al. (Org.). Educação Física esporte e sociedade: temas emergentes. São Cristóvão, ES: Universidade Federal do Espírito Santo, 2008. 
SANTOS, W. dos et al. Avaliação na educação física escolar: reconhecendo a especificidade de um componente curricular. Movimento, Porto Alegre, v. 21, n. 1, p. 205-218, jan./mar. 2015.

SANTOS, W. dos et al. A relação dos alunos com os saberes nas aulas de educação física. Journal Physical Education. Maringá, PR, v. 27, n. 1, p. 1-17, fev. 2016a.

SANTOS, W. dos et al. Significance of assessment experiences during initial teacher training in physical education. Motriz, Rio Claro, SP, v. 22, n. 1, p. 62-71, jan./mar. $2016 \mathrm{~b}$.

SANTOS, W. dos et al. A constituição de um corpus de saberes teóricos e práticos para o ensino da avaliação. In: SANTOS, W. dos (Org.). Avaliação na Educação Física: diálogos com a formação inicial do Brasil, Colômbia, Uruguai e Espanha. São Paulo: Appris, 2018.

SANTOS, W. dos; MAXIMIANO, F. de L.; FROSSARD, M. L. Narrativas docentes sobre avaliação do ensino-aprendizagem: da formação inicial ao contexto de atuação profissional. Movimento, Porto Alegre, v. 22, p. 739-752, jul./set. 2016.

SARNI, M. La evaluación en educación física escolar. 2006. 152 f. Disertación (Maestría en Educación. Énfasis en curriculum y evaluación) - Programa de Posgrado em Educación, Universidad Católica del Uruguay, Montevideo, 2006.

SHAW, I. La evaluación cualitativa. Buenos Aires: Paidos, 2003.

VALDIVIELSO, F. N. Entrenamiento adaptado a los jóvenes. Revista de Educacíon, Mar del Plata, n. 335, p. 61-80, jun. 2004.

VENEZUELA. Ley Orgánica de Educación. Caracas, 1980. Disponível em: $<$ http://www.oas.org/juridico/spanish/mesicic2_ven_anexo_33_sp.pdf>. Acesso em: 22 jan. 2018.

VENEZUELA. Ministerio de Educación. Orientaciones generales para la sistematización en el marco del acompañamiento y seguimento de la educación bolivariana. Caracas: Ministerio de Educación, 2005.

VIANNA, H. M. Avaliação educacional: teoria-planejamento-modelos. São Paulo: Ibrasa, 2000.

WEINECK, J. Entrenamiento óptimo. Madrid: Hispano Europea, 1988.

Recebido em: 26 FEVEREIRO 2018

Aprovado para publicação em: 22 MAlO 2018 
